Juan Benjamin Duarte Duarte* Katherine Julieth Sierra Suárez** Victor Alfonso Rueda Ortiz****

Universidad Industrial de Santander, Bucaramanga, Colombia.

Recibido: 30 de enero de 2015

Concepto de evaluación: 11 de mayo de 2015

Aprobado: 04 de junio de 2015

Artículo de investigación (C) 2015 Universidad Católica de Colombia. Facultad de Ciencias Económicas y Administrativas. Todos los derechos reservados

* Ingeniero industrial. Doctor en Finanzas de Empresas. Profesor titular de la Universidad Industrial de Santander, Facultad de Ingenierías FísicoMecánicas, Escuela de Estudios Industriales y Empresariales, Bucaramanga, Colombia. Dirección de correspondencia: carrera 27 con calle 9, Ciudad Universitaria, Edificio de Ingeniera Industrial, oficina 20725, Bucaramanga, Colombia. Correo electrónico: jbduated@hotmail.com

** Ingeniera industrial. Magíster en Ingeniería Industrial. Profesora becaria de la Universidad Industrial de Santander, Facultad Ingenierías

Físico-Mecánicas, Escuela de Estudios Industriales y Empresariales, Bucaramanga, Colombia. Dirección de correspondencia: carrera 27 con calle 9, Ciudad Universitaria, Edificio de Ingeniera Industrial, oficina 207-

25, Bucaramanga, Colombia. Correo electrónico: katjulss@gmail.com
Finanz. polit. econ., ISSN: 2248-6046, Vol. 7, No. 2, julio-diciembre, 2015, pp. 341-357

http://dx.doi.org/10.14718/revfinanzpolitecon.2015.7.2.7

\section{Análisis comparativo de eficiencia entre Brasil, México y Estados Unidos*}

\section{RESUMEN}

Este artículo busca contrastar la eficiencia débil de los índices bursátiles de Brasil, México y Estados Unidos, desde el supuesto de que un mercado eficiente no es predecible. Con este propósito se evalúa la predictibilidad usando la prueba de rachas y el ratio de varianza automático, en el periodo 1995-2014. Los resultados evidencian que los mercados accionarios de Brasil y México han pasado de ser no eficientes a eficientes en los últimos años; en contraste, Estados Unidos muestra predictibilidad en distintos intervalos de tiempo.

Palabras clave: test de rachas, ratio de varianza automático, eficiencia de mercados.

JEL: C01, C22, G14

\section{Comparative efficiency analysis between Brazil, Mexico and The United States}

\section{ABSTRACT}

This article seeks to contrast the weak form efficiency of the Brazilian, US, and Mexican stock indexes, based on the assumption that an efficient market is not predictable. With this goal in mind, we assessed predictability using runs tests and automatic variance ratio, in the 1995-2014 period. The results shed light on the fact that, in recent years, stock markets in Brazil and Mexico have gone from being non-efficient to being efficient. In contrast, the United States shows predictability at different time intervals.

Keywords: runs test, automatic variance ratio, market efficiency.

Este artículo constituye los resultados parciales del trabajo de investigación Verificación de la hipótesis de mercado adaptativo en Latinoamérica, liderado por la ingeniera Katherina Julieth Sierra Sánchez para optar al título de magíster en Ingeniería Industrial por la Universidad Industrial de Santander, Bucaramanga, Colombia. 


\section{Análise comparativa de eficiência entre Brasil, México e Estados Unidos}

*** Técnico industrial y de producción. Estudiante en Tecnología en Análisis y Desarrollo de Sistemas de Información del Servicio Nacional de Aprendizaje (SENA). Desarrollador de software libre. Dirección de correspondencia: carrera 27 con calle 9, Ciudad Universitaria, Edificio de Ingeniera Industrial, oficina 207-25, Bucaramanga, Colombia. Correo electrónico: ingeruedarortiz@gmail.com

\section{RESUMO}

Este artigo pretende contrastar a fraca eficiência dos índices das bolsas do Brasil, México e Estados Unidos a partir da hipótese de que um mercado eficiente não é previsível. Com esse propósito, avalia-se a previsibilidade usando o teste de aleatoriedade ou rácio da variância automático no período 1995-2014. Os resultados demonstram que os mercados acionários do Brasil e do México passaram de ser não eficientes a eficientes nos últimos anos; em compensação, os Estados Unidos mostram previsibilidade em diferentes intervalos de tempo.

Palavras-chave: teste de aleatoriedade, rácio da variância automático, eficiência de mercados. 


\section{INTRODUCCIÓN}

El premio nobel en economía del 2013 fue otorgado a los investigadores Eugene Fama, Lars Peter Hansen y Robert Shiller, y con ello se evidenció que aún en pleno siglo XXI, el estudio de los mercados financieros sigue siendo un tema de gran relevancia. Cabe destacar que este premio tuvo la particularidad de haber sido entregado a dos estudiosos cuyas teorías se han considerado "opuestas" y que además han liderado el debate acerca de si los mercados son eficientes o conductistas. Esta discusión tiene sus orígenes en 1970, cuando Fama enunció la teoría de mercados eficientes, que se basa en la racionalidad de los agentes del mercado y la aleatoriedad de los precios de los activos; mientras que a finales del siglo XX, Shiller se consolidó como uno de los representantes más influyentes de las finanzas conductuales, que hablan de agentes irracionales que actúan desde sus propios intereses y producen desviaciones anormales en los precios de los activos financieros. De hecho, uno de sus aportes más importantes de Shiller fue la predicción de la burbuja dot-com en el 2000 y de la crisis de las subprime en el 2005, con lo su cual demostró que en dichos periodos no se cumplían los supuestos de la eficiencia.

Ambas teorías han sido estudiadas de forma empírica con distintos resultados de rechazo y aceptación, sin que exista una teoría consolidada y mundialmente aceptada. Con el propósito de contribuir a esta temática, se evalúa la eficiencia de los mercados accionarios de Brasil y México mediante la prueba de rachas y el ratio automático de varianza (AVR, por sus siglas en ingles). Estos países latinoamericanos cobran importancia por el reciente incremento en su inversión directa extranjera (Comisión Económica para América Latina y el Caribe [Cepal], 2011). Para este fin, se usarán los principales índices bursátiles de los mercados estudiados, en función de identificar si han sido eficientes durante los últimos veinte años. Además, se analiza el comportamiento de estos índices en contraste con los principales índices de Estados Unidos: el Standard \& Poor's 500 (S\&P500) y el
Dow Jones Industrial Average (DJIA), dado que el mercado estadounidense ha sido generalmente aceptado como eficiente.

Este artículo se divide en cuatro secciones, estructuradas así: se inicia con la revisión de la literatura acerca del estudio de los mercados financieros, seguida de la metodología estructurada en tres etapas (estadístico descriptivo, pruebas de eficiencia y análisis relativo); posteriormente se hace la interpretación de resultados y finalmente se presentan las conclusiones.

\section{REVISIÓN DE LITERATURA}

En un mercado eficiente, la información está libremente disponible para los participantes, quienes intentan predecir el comportamiento futuro de los precios del mercado, lo cual causa que en un instante de tiempo los precios reflejen toda la información relevante para los inversores con base en eventos ocurridos y en eventos que, se espera, tendrán lugar en el futuro (Fama, 1965). En otras palabras, en un mercado eficiente, el precio actual del activo es un buen estimador de su valor intrínseco y solo varía debido al ingreso aleatorio de nueva información al mercado, lo cual hace imposible su pronóstico sistemático. Sin embargo, Shiller (2003) asegura que los inversores no siempre son racionales, sino que toman decisiones influenciados por la psicología y la sociología. A causa de esto, en el mercado se producen tendencias, burbujas, crisis, entre otros fenómenos que no son explicados por la hipótesis de mercados eficientes y que podrían permitir la predicción y obtención de rendimientos sistemáticos por parte de los inversores que sepan explotar las anomalías del mercado.

Teniendo en cuenta que la eficiencia de los mercados forma parte de los supuestos básicos de los modelos de valoración de activos, tales como el CAPM (Capital Asset Pricing Model) y el APT (Arbitrage Pricing Theory), su rechazo en un mercado bursátil podría indicar la invalidez de estos modelos. Además, en un mercado no eficiente no habría garantía de que se están 
dando las condiciones de un juego justo, lo cual generaría desconfianza en los inversores (Duarte y Mascareñas, 2014).

En cuanto a los resultados empíricos, se han encontrado posibilidades de predicción sistemática, ya sea usando análisis técnico en mercados considerados eficientes como Estados Unidos (Brock, Lakonishok y Lebaron, 1992), Madrid (Fernández y González, 2000) y Canadá (Alexeev y Tapon, 2011); sin embargo, estos resultados son aclarados por otros en los que se concluye que a pesar de encontrarse estructuras de corto y largo plazo en las series de precios que podrían predecir el mercado, los beneficios que generarían se perderían al incluirse los costos de transacción, y con ello se haría imposible ganarle sistemáticamente al mercado (Lu, Shiu y Liu, 2012).

Por otra parte, en cuanto a los estudios realizados en Latinoamérica, Urrutia (1995) fue uno de los primeros en comprobar la aleatoriedad de mercados bursátiles. En su trabajo testea los mercados de Argentina, Chile y México para el periodo 1975-1991, mediante pruebas de rachas y razón de varianzas. Llega así a la conclusión de que estos mercados no son eficientes. Por otra parte, Ojah y Karemera (1999) usan razón de varianza y modelos autorregresivos en los mercados de Argentina, Brasil, Chile y México, en un periodo diferente pero traslapado con el del estudio de Urrutia (1987 a 1997); destacan que sus resultados no evidencian rechazo a la eficiencia. Ese mismo año, Grieb y Reyes (1999) examinan las propiedades de caminata aleatoria de las acciones de Brasil y México mediante el ratio de varianza; encuentran que para Brasil no se rechaza la hipótesis de aleatoriedad, mientras que el mercado mexicano muestra reversión a la media. Años más tarde, Valdivieso (2004) analiza la eficiencia del mercado de México en el periodo 1994-1999, para lo cual usa herramientas estadísticas como correlogramas, el test $\mathrm{Q}$ de Box-Pierce, el test Ljung-Box y el test de rachas; el autor rechaza que el mercado siga una caminata aleatoria.

Por otro lado, Tabak (2007) y Freitas, De Souza y de Almeida (2009) evalúan el mercado de
Brasil mediante raíces unitarias y modelos de predicción, respectivamente; concluyen que este mercado no es eficiente durante el periodo 1998-2007. Además, Butler (2012) estudia diversos mercados del mundo, entre ellos Brasil, México y Argentina, durante el periodo 2000-2010; sus resultados establecen que en todos los mercados considerados hubo periodos en los que se presentaba tendencia estacionaria, mientras que en otros no, con lo cual se demostró que la eficiencia no es permanente en estos mercados.

Por su parte, Inghelbrecht et al. (2012) evaluaron las técnicas de negociación que usan los inversionistas en diversos mercados emergentes, incluyendo Argentina, Brasil, Colombia, Chile, Ecuador, México y Venezuela. Estos autores concluyeron que cuando se ajustan las reglas a los costos de negociación, no es posible superar la estrategia de comprar y mantener; sin embargo, durante la crisis del 2008 aparecieron ineficiencias en varios mercados, lo cual demostró la influencia de condiciones externas en la eficiencia de los rendimientos de las acciones.

Recientemente, Duarte y Mascareñas (2014) evaluaron el cumplimiento de la caminata aleatoria en los mercados bursátiles de Latinoamérica; haIlaron así una mejora en la eficiencia de los activos de México, Brasil y Colombia desde 2007 hasta 2012, mientras que observaron un menor nivel de aleatoriedad en Perú y Chile con respecto a sus pares latinoamericanos durante todo el periodo de estudio (2002-2012).

Por otra parte, Duarte y Mascareñas (2012) realizan una revisión exhaustiva de la literatura empírica relacionada con la eficiencia de mercados y encuentran que el $60 \%$ de ella niega la existencia de mercados eficientes, mientras que solo el $5 \%$ acepta un incremento del nivel de aleatoriedad en las bolsas durante la última década del siglo XX y principios del siglo XXI. Debido a la aparición de resultados mixtos en la literatura revisada referente a la aleatoriedad de los mercados de Brasil y México, este artículo busca evaluar la eficiencia de los principales índices bursátiles que los representan, mediante pruebas de caminata aleatoria, 
como rachas y ratio de varianza automático en los últimos veinte años.

\section{METODOLOGÍA}

\section{Datos}

En este trabajo se analiza la serie del Índice de la Bolsa de Valores del Estado de São Paulo (IBOVESPA), que es el más representativo del mercado bursátil de Brasil, además del Índice de Precios y Cotizaciones (IPC), principal índice accionario del mercado de México. Ambos índices son analizados en un periodo de 20 años, desde el 2 de enero de 1995 hasta el 31 de diciembre del 2014. Se omiten los días en los que la bolsa no operó y los que presentan valores nulos. La serie se analiza con valores diarios en su periodo completo y usando diez subperiodos de 2 años (aproximadamente 500 datos), con el fin de evaluar la evolución de la eficiencia débil de los mercados objeto de estudio. Además, con el propósito de hacer una evaluación de la eficiencia relativa de los mercados de Brasil y México, se toman como referentes a los índices Standard and Poor's 500 (S\&P500) y Dow Jones Industrial Average (DJIA), que son los más representativos de Estados Unidos. Las series de cotizaciones son tomadas de la base de datos Bloomberg, y se utilizan como variable de estudio la rentabilidad continua de cada activo.

\section{Etapas}

La metodología por utilizar se compone de tres etapas secuenciales. La primera consiste en la estimación de los estadísticos preliminares de las series de retornos por evaluar (IBOVESPA, IPC, SP\&500 y DJIA), para identificar sus principales características econométricas y su aproximación a una distribución normal.

En la segunda etapa se contrasta la eficiencia débil de las bolsas de Brasil, México y Estados Unidos, desde el supuesto de que un mercado eficiente no es predecible a partir de su serie de precios históricos. Con este propósito, se usan la prueba de rachas y el test ratio de varianza automático, definidos a continuación.

La prueba de rachas analiza el número total de rachas que se presentan en una serie; si esta cantidad es muy pequeña o muy grande, es indicio de baja aleatoriedad en los datos, lo que conlleva el rechazo de la hipótesis nula de que los retornos son independientes e idénticamente distribuidos (i.i.d.). Este test se determina mediante la estimación del número de rachas con datos superiores a la media de los retornos $\left(\mathrm{n}_{1}\right)$ y la cantidad de rachas con valores inferiores a la media $\left(n_{2}\right)$, cuya suma genera el número total de rachas $(R)$; variable que se comporta asintóticamente como una distribución normal y que puede ser estandarizada mediante el estadístico Z:

$\mathrm{Z}=\frac{\mathrm{R}-\hat{\mu}}{\hat{\sigma}}$, donde $\hat{\mu}=\left[\frac{2 * \mathrm{n}_{1} * \mathrm{n}_{2}}{\mathrm{n}_{1}+\mathrm{n}_{2}}\right]+1, \mathrm{y}$

$\sigma=\sqrt{\frac{\left(2 * \mathrm{n} 1 * \mathrm{n} 2 *\left(2 * \mathrm{n} 1 * \mathrm{n} 2-\mathrm{n}_{1}-\mathrm{n}_{2}\right)\right)}{\left(\left(\mathrm{n}_{1}+\mathrm{n}_{2}\right)^{2} *\left(\mathrm{n}_{1}+\mathrm{n}_{2}-1\right)\right)}}$

Se rechaza la hipótesis de retornos i.i.d. si el P-valor es inferior al nivel de confianza.

Asimismo, se utiliza una segunda prueba para contrastar la eficiencia débil, conocida como el test ratio de varianza automático, planteado inicialmente por Choi (1999) y modificado por Kim (2009), para detectar correlaciones seriales que podrían dar indicios de predictibilidad en un mercado. Este test es explicado por Ely (2011), quien se basa en el trabajo de Lo y MacKinlay (1988) para estimar el ratio de varianza de una serie $\left(Y_{t}\right)$ mediante esta ecuación:

$V R(k)=1+2 \sum_{J=1}^{T-1} m\left(\frac{i}{k}\right) \hat{p}_{i}$

Donde $\hat{p} i$ es el estimador del coeficiente de autocorrelación de orden $i$-ésimo, y k es el periodo holding (de referencia), mientras que $m(i / k)$ es la función cuadrática espectral dada por la expresión:

$m\left(\frac{i}{k}\right)=\frac{25}{12 \pi^{2}\left(\frac{i}{k}\right)^{2}}\left[\frac{\sin \left(\frac{6 \pi}{5}\right)}{\frac{6 \pi \frac{i}{k}}{5}}-\cos \left(\frac{6 \pi \frac{i}{k}}{5}\right)\right]$ 
La hipótesis nula del ratio de varianza plantea que $V R(k)=1$, lo que significaría que no se detecta autocorrelación en el rezago i-ésimo para todo $k$. La elección de $k$ se hace generalmente de forma arbitraria; no obstante, Choi (1999) propone un método dependiente solamente de los datos para la elección de $\hat{k}$ óptimo, mediante esta ecuación:

$\hat{k}=1,3221(\hat{\alpha}(2) * T)^{1 / 5}$

Donde $T$ es el tamaño de muestra y $\hat{\alpha}(2)$ depende de la autocorrelación de primer orden $\hat{p}(1)$ :

$\hat{\alpha}(2)=\frac{4 \hat{\rho}(1)^{2}}{(1-\hat{\rho}(1))^{4}}$

A partir del ratio de varianza de la ecuación [2] y el parámetro $\widehat{k}$ (periodo holding) de la ecuación [4], se estima el ratio de varianza automático (AVR), mediante la ecuación [6]:

$A V R(\hat{k})=\frac{\sqrt{\frac{T}{\hat{k}}}[V R(\hat{k})-1]}{\sqrt{2}} \stackrel{d}{\rightarrow} N(0,1)$

El estadístico AVR $(\widehat{k})$ desde el supuesto de que los retornos son independientes e idénticamente distribuidos (i.i.d.), se distribuye normalmente con media 0 y varianza 1 . Sin embargo, cuando existe heterocedasticidad, la inferencia estadística puede ser inválida en pequeñas muestras, razón por la cual Kim (2009) propone una técnica bootstrap que provee inferencia estadística adecuada en pequeñas muestras bajo heterocedasticidad condicional. El proceso bootstrap consiste en:

a) Formar una muestra bootstrap de $T$ observaciones $Y_{t}^{*}=n_{t} Y_{t}(t=1, \ldots, T)$, donde $Y_{t}$ es la serie original y $n_{t} \sim N(0,1)$ es una secuencia aleatoria que sigue una distribución normal estándar.

b) Calcular el estadístico $A V R^{*}\left(\widehat{k}^{*}\right)$ a partir de la serie formada anteriormente $\left\{Y_{t}^{*}\right\}_{t=1}^{T}$.

c) Repetir los dos pasos anteriores 500 veces para formar una distribución boots$\operatorname{trap}\left\{A V R^{*}\left(\hat{k}^{*} ; j\right)\right\}_{j=1}^{500}$.

La distribución bootstrap construida se aproxima a la distribución del estadístico AVR (ver ecuación [6]) si se cumple la hipótesis nula de aleatoriedad y no autocorrelación en los retornos, por lo cual al estimar el ratio de varianza automático de la serie original $\left(Y_{t}\right)$ se rechaza la hipótesis nula cuando el estadístico cae fuera del intervalo de confianza $(1-\alpha) \%$, construido mediante la ecuación [7]:

$$
\left\{A V R^{*}(\alpha / 2), A V R^{*}(1-\alpha / 2),\right\}
$$

Donde $\operatorname{AVR}^{*}(\alpha / 2)$ denota el percentil $\alpha / 2$ de la distribución bootstrap $\left\{A V R^{*}\left(\hat{k}^{*} ; j\right)\right\}_{j=1}^{500}$. Asimismo, $A V R^{*}(1-\alpha / 2)$ es el percentil $1-\alpha / 2$ de esta distribución.

Finalmente, en la tercera etapa se lleva a cabo la comparación de la eficiencia de los mercados de Brasil y México versus el de Estados Unidos.

\section{RESULTADOS}

\section{Estadísticas preliminares}

En la tabla 1 se presentan las estadísticas básicas de las series de retornos del IBOVESPA, IPC, S\&P500 y DJIA.

Tabla 1.

Estadísticos descriptivos

\begin{tabular}{|c|c|c|c|c|}
\hline & IBOVESPA & IPC & S\&P 500 & DJIA \\
\hline Media & 0,0005 & $0,0006 * * *$ & $0,0003^{*}$ & $0,0003^{*}$ \\
\hline Mediana & 0,0010 & 0,0008 & 0,0007 & 0,0006 \\
\hline Máximo & 0,2882 & 0,1215 & 0,1096 & 0,1051 \\
\hline Mínimo & $-0,1723$ & $-0,1431$ & $-0,0947$ & $-0,0820$ \\
\hline $\begin{array}{c}\text { Desviación } \\
\text { estándar }\end{array}$ & 0,0220 & 0,0153 & 0,0122 & 0,0115 \\
\hline $\begin{array}{c}\text { Sesgo } \\
\text { Curtosis }\end{array}$ & 16,2476 & 9,7875 & 11,1129 & 10,9308 \\
\hline $\begin{array}{c}\text { Jarque Bera } \\
\text { arqu }\end{array}$ & $36367 * * *$ & $9653^{* * *}$ & $13862 * * *$ & $13220 * * *$ \\
\hline $\begin{array}{c}\text { Numero de } \\
\text { datos }\end{array}$ & 4949 & 5027 & 5036 & 5036 \\
\hline
\end{tabular}

*Significativo al 10\%; *** significativo al $1 \%$.

Fuente: elaboración de los autores a partir de los paquetes estadísticos Eviews y SPSS. 
Las estadísticas evidencian que aunque todas las series presentan medias positivas, a un nivel de $5 \%$ de significancia, solamente la media del IPC puede considerarse estadísticamente significativa. Además, al comparar las series, se observa que los estadísticos como mediana, máximo y mínimo son muy similares, aunque hay una mayor desviación estándar en la serie del IBOVESPA. La principal diferencia se presenta en el sesgo que indica que los datos de Brasil y México se agrupan hacia la izquierda de la media, por lo cual es menor la probabilidad de obtener ganancias significativas en comparación con las series del S\&P 500 y del DJIA, cuyos sesgos son negativos. Por último, se detecta que todas las series son leptocúrticas, y la significancia del estadístico Jarque-Bera rechaza la hipótesis de que los retornos sigan una distribución normal.

\section{Test de eficiencia débil}

\section{Brasil}

La eficiencia del mercado de Brasil es evaluada con la serie del IBOVESPA mediante la prueba de rachas y el ratio de varianza automático.
En la tabla 2 se presentan los resultados del test de rachas en la serie del IBOVESPA, donde se detecta que no es posible rechazar la hipótesis de aleatoriedad de la serie durante el periodo completo 1995-2014. No obstante, al analizar los subperiodos de dos años, se evidencia que entre los años 1997 y 1998 (subperiodo 2), la serie del IBOVESPA no cumple con la hipótesis de retornos i.i.d., lo que indicaría la existencia de patrones lineales en la serie, lo cual da lugar a una posible predictibilidad del índice en estos años.

Con el propósito de verificar el resultado anterior, se estima el estadístico AVR en el periodo completo y los diez subperiodos de dos años. Los resultados son presentados en la tabla 3 con su respectivo P-valor. Adicionalmente, en la gráfica 1 se grafica el comportamiento del estadístico AVR en los diez subperiodos con respecto a un intervalo de confianza del $95 \%$.

De la tabla 3 se deduce que el mercado de Brasil ha sido eficiente en forma débil durante el periodo completo de estudio, lo cual permite corroborar lo hallado mediante la prueba de rachas. Asimismo, en la gráfica 1 se observa que a un nivel de confianza del $95 \%$ no puede rechazarse la hipótesis de aleatoriedad o no correlación serial en ninguno de los diez subperiodos estudiados,

Tabla 2.

Test de rachas en el IBOVESPA

\begin{tabular}{|c|c|c|c|c|c|c|c|c|}
\hline \multicolumn{3}{|c|}{ Periodo } & Media & $\begin{array}{c}\text { Casos }< \\
\text { media }\end{array}$ & $\begin{array}{c}\text { Casos }>= \\
\text { media }\end{array}$ & R & Z & P-valor (\%) \\
\hline Completo & $02 / 01 / 1995$ & $30 / 12 / 2014$ & 0,0005 & 2409 & 2540 & 2463 & $-0,31$ & 75,9 \\
\hline 1 & $02 / 01 / 1995$ & $03 / 01 / 1997$ & 0,0010 & 244 & 251 & 244 & $-0,40$ & 68,9 \\
\hline 2 & $06 / 01 / 1997$ & $05 / 01 / 1999$ & 0,0000 & 219 & 276 & 215 & $-2,76 * * *$ & 0,6 \\
\hline 3 & $06 / 01 / 1999$ & $04 / 01 / 2001$ & 0,0017 & 255 & 240 & 249 & 0,07 & 94,8 \\
\hline 4 & $05 / 01 / 2001$ & $06 / 01 / 2003$ & $-0,0007$ & 250 & 245 & 244 & $-0,40$ & 68,7 \\
\hline 5 & $07 / 01 / 2003$ & $29 / 12 / 2004$ & 0,0016 & 237 & 258 & 242 & $-0,55$ & 58,5 \\
\hline 6 & $30 / 12 / 2004$ & $27 / 12 / 2006$ & 0,0011 & 245 & 250 & 251 & 0,23 & 82,0 \\
\hline 7 & $28 / 12 / 2006$ & $30 / 12 / 2008$ & $-0,0003$ & 222 & 273 & 254 & 0,74 & 46,0 \\
\hline 8 & $02 / 01 / 2009$ & $04 / 01 / 2011$ & 0,0013 & 243 & 252 & 237 & $-1,03$ & 30,4 \\
\hline 9 & $05 / 01 / 2011$ & $03 / 01 / 2013$ & $-0,0002$ & 249 & 246 & 253 & 0,41 & 68,5 \\
\hline 10 & $03 / 01 / 2013$ & $30 / 12 / 2014$ & $-0,0005$ & 251 & 243 & 257 & 0,82 & 41,4 \\
\hline
\end{tabular}

*** Significativo al 1\%; R: número de rachas; Z: estadístico (ecuación [1]); P-valor: significancia estadístico Z. Fuente: elaboración de los autores a partir del paquete estadístico SPSS. 
Tabla 3.

Estadísticos AVR en el IBOVESPA

\begin{tabular}{|c|c|c|c|c|c|c|}
\hline \multicolumn{3}{|c|}{ Periodo } & $k$ & $\begin{array}{c}\text { Desviación } \\
\text { estándar }\end{array}$ & AVR & P-valor (\%) \\
\hline Completo & $\mathbf{0 2 / 0 1 / 1 9 9 5}$ & $\mathbf{3 0 / 1 2 / 2 0 1 4}$ & $\mathbf{2 , 3}$ & $\mathbf{0 , 4 3 0 5}$ & $\mathbf{0 , 6 4 2 7}$ & $\mathbf{1 5 , 0}$ \\
\hline 1 & $02 / 01 / 1995$ & $03 / 01 / 1997$ & 2,5 & 0,4238 & 0,5665 & 19,8 \\
\hline 2 & $06 / 01 / 1997$ & $05 / 01 / 1999$ & 1,7 & 0,3592 & 0,0878 & 59,2 \\
\hline 3 & $06 / 01 / 1999$ & $04 / 01 / 2001$ & 1,5 & 0,4433 & 0,0173 & 86,6 \\
\hline 4 & $05 / 01 / 2001$ & $06 / 01 / 2003$ & 1,5 & 0,1768 & $-0,0055$ & 91,6 \\
\hline 5 & $07 / 01 / 2003$ & $29 / 12 / 2004$ & 1,5 & 0,2128 & $-0,0216$ & 74,4 \\
\hline 6 & $30 / 12 / 2004$ & $27 / 12 / 2006$ & 2,1 & 0,1582 & $0,3257^{*}$ & 7,2 \\
\hline 7 & $28 / 12 / 2006$ & $30 / 12 / 2008$ & 1,4 & 0,3005 & 0,0394 & 73,2 \\
\hline 8 & $02 / 01 / 2009$ & $04 / 01 / 2011$ & 1,7 & 0,2043 & $-0,0621$ & 48,2 \\
\hline 9 & $05 / 01 / 2011$ & $03 / 01 / 2013$ & 1,2 & 0,2438 & 0,0916 & 41,6 \\
\hline 10 & $03 / 01 / 2013$ & $30 / 12 / 2014$ & 1,8 & 0,2079 & $-0,1394$ & 25,8 \\
\hline
\end{tabular}

* Significativo al 10\%; k: periodo holding (ecuación [5]); P-valor: significancia del estadístico AVR.

Fuente: elaboración de los autores a partir de MATLAB.

ni siquiera en el subperiodo 2, que fue evaluado como no aleatorio mediante la prueba de rachas.

De la gráfica 1 se observa que el intervalo de confianza fue más amplio durante los tres primeros subperiodos (1995 a 2001), lo que podría indicar una mayor incertidumbre en el mercado, es decir, una mayor aleatoriedad en las rentabilidades de los activos. También se observa que el estadístico AVR se mantiene cercano a 0 , y solamente en el subperiodo 6 (2004 a 2006) podría considerarse positivo y significativo a un $10 \%$ de confianza (tabla 3 ), lo que indicaría que durante este subperiodo podría haber existido correlación positiva en la serie, es decir, un mantenimiento de las tendencias. Es importante resaltar que este intervalo de tiempo está caracterizado por ser el periodo precrisis de la subprime.

\section{México}

Al igual que el mercado de Brasil, México se evalúa con las pruebas de rachas y el ratio de varianza

Gráfica 1 .

Estadístico AVR en el IBOVESPA con intervalo de confianza al 95\%

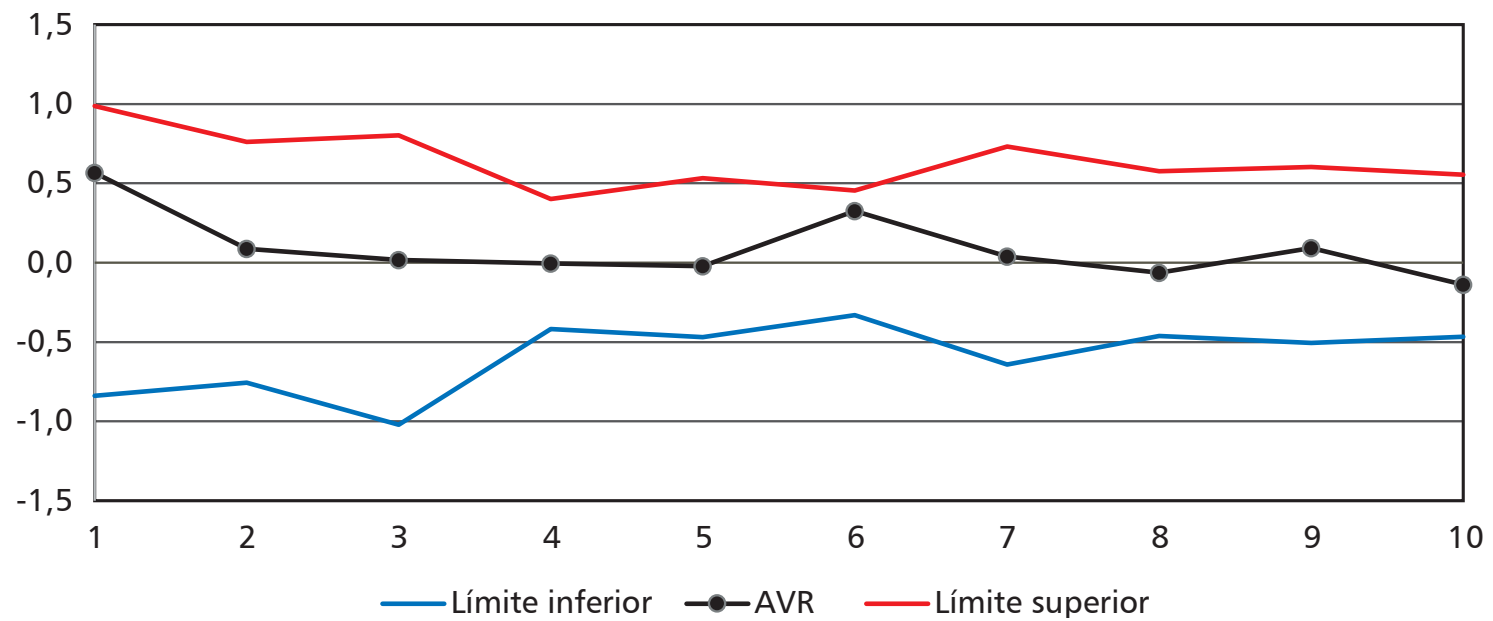

Nota: en la gráfica se observan los valores del estadístico AVR para diez subperiodos de tiempo (línea negra: subperiodos de la tabla 2) y los límites inferior y superior al 95\% de confianza (líneas grises) para la serie del IBOVESPA. Fuente: elaboración de los autores a partir de MATLAB. 
Tabla 4.

Test de rachas en el IPC

\begin{tabular}{|c|c|c|c|c|c|c|c|c|}
\hline \multicolumn{3}{|c|}{ Periodo } & Media & $\begin{array}{c}\text { Casos < } \\
\text { media }\end{array}$ & $\begin{array}{c}\text { Casos }>= \\
\text { media }\end{array}$ & $\mathrm{R}$ & Z & P-valor (\%) \\
\hline Completo & 03/01/1995 & $31 / 12 / 2014$ & 0,0006 & 2479 & 2548 & 2348 & $-4,68 * * *$ & 0,0 \\
\hline 1 & 03/01/1995 & 24/12/1996 & 0,0007 & 265 & 236 & 218 & $-2,93 * * *$ & 0,3 \\
\hline 2 & 26/12/1996 & $21 / 12 / 1998$ & 0,0003 & 245 & 256 & 216 & $-3,17^{* * *}$ & 0,2 \\
\hline 3 & $22 / 12 / 1998$ & $15 / 12 / 2000$ & 0,0008 & 254 & 247 & 224 & $-2,46^{* *}$ & 1,4 \\
\hline 4 & $18 / 12 / 2000$ & $19 / 12 / 2002$ & 0,0002 & 261 & 240 & 235 & $-1,44$ & 15,0 \\
\hline 5 & $20 / 12 / 2002$ & $17 / 12 / 2004$ & 0,0014 & 241 & 260 & 231 & $-1,80^{*}$ & 7,1 \\
\hline 6 & $20 / 12 / 2004$ & $14 / 12 / 2006$ & 0,0014 & 238 & 263 & 235 & $-1,42$ & 15,5 \\
\hline 7 & $15 / 12 / 2006$ & $12 / 12 / 2008$ & $-0,0004$ & 233 & 268 & 239 & $-1,01$ & 31,1 \\
\hline 8 & $15 / 12 / 2008$ & $10 / 12 / 2010$ & 0,0011 & 234 & 267 & 244 & $-0,58$ & 56,5 \\
\hline 9 & $13 / 12 / 2010$ & $10 / 12 / 2012$ & 0,0002 & 248 & 253 & 248 & $-0,31$ & 75,6 \\
\hline 10 & $11 / 12 / 2012$ & $31 / 12 / 2014$ & 0,0000 & 264 & 254 & 268 & 0,71 & 47,6 \\
\hline
\end{tabular}

* Significativo al 10\%; ** significativo al 5\%; *** significativo al 1\%; R: número de rachas; Z: estadístico (ecuación [1]); P-valor: significancia estadístico Z.

Fuente: elaboración de los autores a partir del paquete SPSS.

automático en el índice más representativo de su mercado accionario, como es el IPC.

En la tabla 4 se presentan los resultados obtenidos mediante la prueba de rachas en las rentabilidades del IPC. Se observa que en el periodo completo (1995 a 2014) se rechaza la hipótesis de que los retornos sean independientes e idénticamente distribuidos. Sin embargo, al realizar análisis por subperiodos, se encuentra que este rechazo ocurre principalmente en el periodo 1995-2004, y que en los años siguientes la serie se evalúa como aleatoria. Esto podría interpretarse como una mejora en la eficiencia del mercado de México.

Para corroborar los resultados anteriores, se usa el estadístico AVR, el cual se muestra en la tabla 5 acompañado de su respectivo P-valor para el periodo completo y los diez subperiodos construidos; adicionalmente, en la gráfica 2 se grafica el comportamiento del estadístico AVR en los subperiodos estudiados con respecto a un intervalo de confianza del $95 \%$.

Por su parte, la tabla 5 muestra que, contrario a lo hallado con la prueba de rachas, no

Tabla 5.

Estadísticos AVR en el IPC

\begin{tabular}{|c|c|c|c|c|c|c|}
\hline \multicolumn{3}{|c|}{ Periodo } & k & $\begin{array}{c}\text { Desviación } \\
\text { estándar }\end{array}$ & AVR & P-valor (\%) \\
\hline Completo & $03 / 01 / 1995$ & $31 / 12 / 2014$ & 4,0 & 0,3668 & $-0,5980$ & 13,4 \\
\hline 1 & $03 / 01 / 1995$ & $24 / 12 / 1996$ & 3,3 & 0,3340 & 0,5377 & 12,2 \\
\hline 2 & $26 / 12 / 1996$ & $21 / 12 / 1998$ & 1,0 & 0,3758 & $-0,0382$ & 76,6 \\
\hline 3 & $22 / 12 / 1998$ & $15 / 12 / 2000$ & 3,2 & 0,1682 & $0,4240^{* *}$ & 3,4 \\
\hline 4 & $18 / 12 / 2000$ & $19 / 12 / 2002$ & 2,4 & 0,2076 & 0,3546 & 12,0 \\
\hline 5 & $20 / 12 / 2002$ & $17 / 12 / 2004$ & 2,5 & 0,2077 & $0,4640^{*}$ & 6,6 \\
\hline 6 & $20 / 12 / 2004$ & $14 / 12 / 2006$ & 3,0 & 0,2045 & $0,6905^{* *}$ & 1,8 \\
\hline 7 & $15 / 12 / 2006$ & $12 / 12 / 2008$ & 2,3 & 0,3035 & 0,4667 & 14,6 \\
\hline 8 & $15 / 12 / 2008$ & $10 / 12 / 2010$ & 2,2 & 0,1886 & $0,3453^{*}$ & 9,8 \\
\hline 9 & $13 / 12 / 2010$ & $10 / 12 / 2012$ & 1,1 & 0,2218 & 0,0747 & 48,2 \\
\hline 10 & $11 / 12 / 2012$ & $31 / 12 / 2014$ & 2,4 & 0,2086 & $0,4496^{*}$ & 6,8 \\
\hline
\end{tabular}

* Significativo al 10\%; ** significativo al 5\%; ${ }^{* *}$ significativo al 1\%; k: periodo holding (ecuación [5]); P-valor: significancia del estadístico AVR.

Fuente: elaboración de los autores a partir de MATLAB. 
puede rechazarse la hipótesis nula de aleatoriedad durante los últimos veinte años. Sin embargo, al hacer un estudio más específico por subperiodos, se evidencia que existen periodos con predictibilidad significativa al 95\% en los intervalos de 1998 a 2000 y de 2004 a 2006.

Por otra parte, en la gráfica 2 se observa que el intervalo de confianza fue más amplio en el primer y segundo subperiodo (1995 a 1998), lo que podría indicar mayor aleatoriedad en las rentabilidades de los activos. En cuanto al estadístico AVR, se evidencia que su magnitud cambia en el tiempo y que hay subperiodos con mayor correlación que otros. Además, solo se muestra correlación negativa en el subperiodo de 1996 a 1998, es decir, la mayor parte de los estadísticos AVR estimados son positivos, lo cual indica que las tendencias en los precios diarios se mantendrían. Es importante resaltar que los subperiodos en los que se detecta predictibilidad al $95 \%$ de confianza mediante el test AVR coinciden con los hallados en el test de rachas; con la particularidad de que se presentan en los periodos precrisis dot-com (1998 a 2000) y precrisis de la subprime (2004 a 2006).

\section{Estados Unidos}

Siguiendo la metodología planteada para los dos mercados anteriores, se utilizan la prueba de rachas y el estadístico AVR en los índices accionarios más representativos del mercado estadounidense, como son el S\&P500 y el DJIA.

En la tablas 6 y 7 se presentan los resultados de la prueba de rachas en los índices del S\&P500 y el DJIA, respectivamente. Se resalta que aunque estos índices son los más representativos del mercado, el primero se compone de las 500 empresas más grandes de Estados Unidos, mientras que el segundo refleja la evolución de las 30 mayores acciones de la bolsa de Nueva York, dejando de lado a empresas gigantescas como Apple. Debido a esto, se considera que el S\&P500 es más amplio al representar más sectores económicos del mercado estadounidense que el índice DJIA. Pese a estas diferencias, los resultados para ambos índices coinciden cuando muestran rechazo a la hipótesis de retornos independientes e idénticamente distribuidos en el periodo completo de estudio (1995 a 2014), mientras que al analizar por subperiodos, se observa que esta aleatoriedad

Gráfica 2 .

Estadístico AVR en el IPC con intervalo de confianza al 95\%

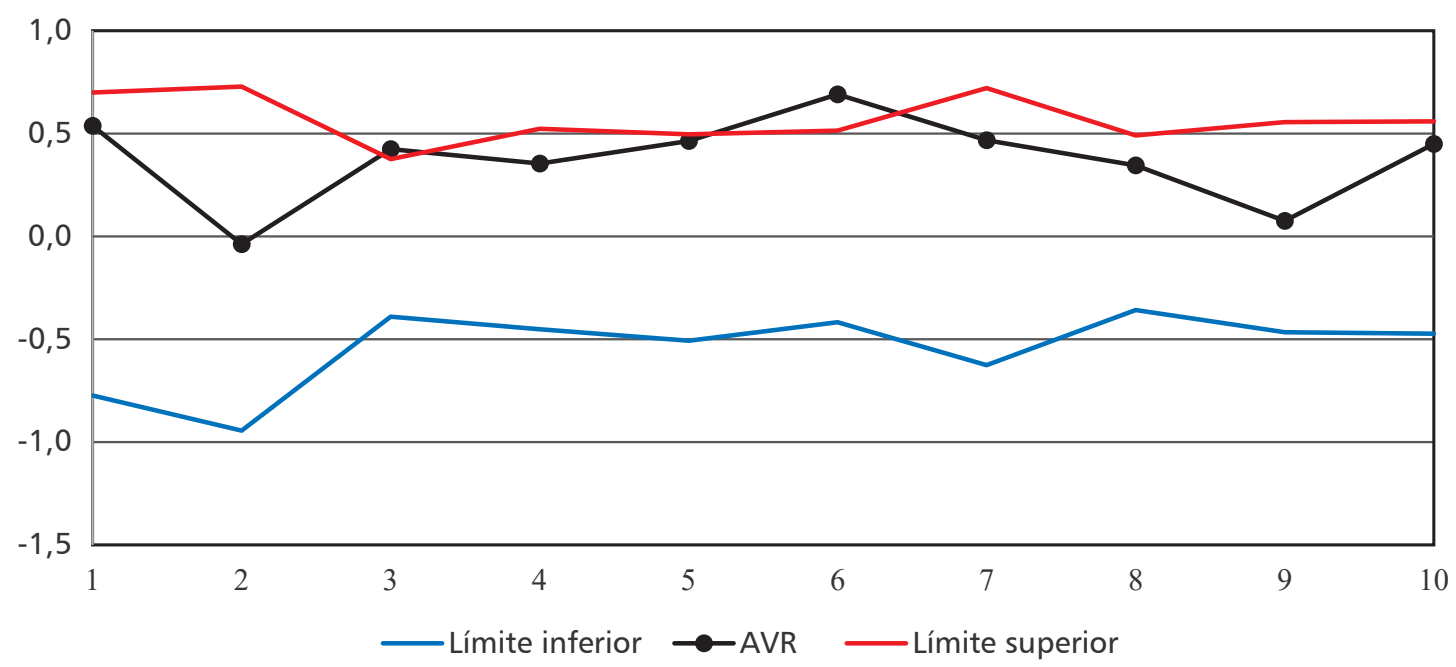

Nota: en la gráfica se observan los valores del estadístico AVR para diez subperiodos de tiempo (línea negra: subperiodos de la tabla 2) y los límites inferior y superior al 95\% de confianza (líneas grises) para la serie del IPC.

Fuente: elaboración de los autores a partir de MATLAB. 
se concentra principalmente en los subperiodos de 2002 a 2004, 2006 a 2008 (inicio crisis de la subprime) y 2012 a 2014 . Es importante destacar que a pesar de que los resultados son similares para los dos índices, entre los años 2000 y 2012, los niveles de significancia del estadístico (P-valor) son mayores en el DJIA que en el S\&P500, lo que se interpreta como mayor aleatoriedad en el Dow Jones, concentrándose en los años de 2004 hasta 2010.
Con el propósito de corroborar los resultados anteriores se estima los estadísticos AVR para los índices S\&P500 y el DJIA, presentados en las tabla 8 y 9 , respectivamente. En estas se corrobora que el mercado no ha sido eficiente durante los últimos veinte años. No obstante, al igual que con la prueba de rachas, se observa que este resultado no es constante durante los diez subperiodos estudiados, sino que la no eficiencia se concentra en intervalos

Tabla 6.

Test de rachas en el SEPP00

\begin{tabular}{|c|c|c|c|c|c|c|c|c|}
\hline \multicolumn{3}{|c|}{} & Media & $\begin{array}{c}\text { Casos } \\
\text { media }\end{array}$ & $\begin{array}{c}\text { Casos }>= \\
\text { media }\end{array}$ & R & Z P-valor (\%) \\
\hline Completo & $03 / 01 / 1995$ & $31 / 12 / 2014$ & 0,0003 & 2406 & 2630 & 2643 & $3,64^{* * *}$ & 0,0 \\
\hline 1 & $03 / 01 / 1995$ & $24 / 12 / 1996$ & 0,0010 & 257 & 245 & 250 & $-0,17$ & 86,8 \\
\hline 2 & $26 / 12 / 1996$ & $21 / 12 / 1998$ & 0,0009 & 240 & 262 & 255 & 0,31 & 75,5 \\
\hline 3 & $22 / 12 / 1998$ & $15 / 12 / 2000$ & 0,0002 & 259 & 243 & 258 & 0,56 & 57,6 \\
\hline 4 & $18 / 12 / 2000$ & $19 / 12 / 2002$ & $-0,0008$ & 256 & 246 & 264 & 1,08 & 27,9 \\
\hline 5 & $20 / 12 / 2002$ & $17 / 12 / 2004$ & 0,0006 & 243 & 259 & 284 & $2,89^{* * *}$ & 0,4 \\
\hline 6 & $20 / 12 / 2004$ & $14 / 12 / 2006$ & 0,0004 & 233 & 269 & 279 & $2,54^{* *}$ & 1,1 \\
\hline 7 & $15 / 12 / 2006$ & $12 / 12 / 2008$ & $-0,0010$ & 217 & 285 & 277 & $2,69^{* * *}$ & 0,7 \\
\hline 8 & $15 / 12 / 2008$ & $10 / 12 / 2010$ & 0,0007 & 237 & 265 & 270 & $1,68^{*}$ & 9,2 \\
\hline 9 & $13 / 12 / 2010$ & $10 / 12 / 2012$ & 0,0003 & 242 & 260 & 236 & $-1,40$ & 16,1 \\
\hline 10 & $11 / 12 / 2012$ & $31 / 12 / 2014$ & 0,0007 & 253 & 265 & 284 & $2,12^{* *}$ & 3,4 \\
\hline
\end{tabular}

* Significativo al 10\%; ${ }^{* *}$ significativo al 5\%; ${ }^{* *}$ significativo al 1\%; R: número de rachas; Z: estadístico (ecuación [1]); P-valor: significancia estadístico Z.

Fuente: elaboración de los autores a partir del paquete SPSS.

Tabla 7.

Test de rachas en el DJIA

\begin{tabular}{|c|c|c|c|c|c|c|c|c|}
\hline \multicolumn{3}{|c|}{} & Media & $\begin{array}{c}\text { Casos }< \\
\text { media }\end{array}$ & $\begin{array}{c}\text { Casos }>= \\
\text { media }\end{array}$ & R & Z & P-valor (\%) \\
\hline Completo & $\mathbf{0 3 / 0 1 / 1 9 9 5}$ & $\mathbf{3 0 / 1 2 / 2 0 1 4}$ & $\mathbf{0 , 0 0 0 3}$ & $\mathbf{2 4 3 0}$ & $\mathbf{2 6 0 6}$ & $\mathbf{2 6 2 6}$ & $\mathbf{3 , 1} \mathbf{1}^{* * *}$ & $\mathbf{0 , 2}$ \\
\hline 1 & $03 / 01 / 1995$ & $02 / 01 / 1997$ & 0,0010 & 247 & 260 & 248 & $-0,56$ & 57,3 \\
\hline 2 & $02 / 01 / 1997$ & $05 / 01 / 1999$ & 0,0007 & 245 & 261 & 243 & $-0,96$ & 33,8 \\
\hline 3 & $05 / 01 / 1999$ & $05 / 01 / 2001$ & 0,0003 & 256 & 250 & 256 & 0,18 & 85,6 \\
\hline 4 & $05 / 01 / 2001$ & $15 / 01 / 2003$ & $-0,0004$ & 259 & 247 & 273 & $1,70^{*}$ & 8,8 \\
\hline 5 & $15 / 01 / 2003$ & $19 / 01 / 2005$ & 0,0004 & 253 & 253 & 287 & $2,94^{* * *}$ & 0,3 \\
\hline 6 & $19 / 01 / 2005$ & $24 / 01 / 2007$ & 0,0004 & 242 & 264 & 266 & 1,11 & 26,6 \\
\hline 7 & $24 / 01 / 2007$ & $27 / 01 / 2009$ & $-0,0009$ & 226 & 280 & 272 & $1,88^{*}$ & 6,0 \\
\hline 8 & $27 / 01 / 2009$ & $28 / 01 / 2011$ & 0,0007 & 254 & 252 & 266 & 1,07 & 28,5 \\
\hline 9 & $28 / 01 / 2011$ & $04 / 02 / 2013$ & 0,0003 & 249 & 257 & 250 & $-0,35$ & 72,6 \\
\hline 10 & $04 / 02 / 2013$ & $30 / 12 / 2014$ & 0,0005 & 231 & 250 & 266 & $2,27^{* *}$ & 2,3 \\
\hline
\end{tabular}

* Significativo al 10\%; ** significativo al 5\%; *** significativo al 1\%; R: número de rachas; Z: estadístico (ecuación [1]); P-valor: significancia estadístico Z.

Fuente: elaboración de los autores a partir del paquete SPSS. 
de tiempo que coinciden entre el S\&P500 y el DJIA, específicamente entre 1995 y 1996 y entre 2006 y 2008. El último subperiodo también es evaluado como no eficiente según los resultados del test de rachas, y a partir de ello se evidencia que la crisis de la subprime pudo haber afectado de forma significativa la eficiencia del mercado de Estados
Unidos. Además, se evidencia menor aleatoriedad en el índice S\&P500 durante el periodo de diciembre de 2002 a diciembre de 2004, resultado similar al de la prueba de rachas.

Con el fin de analizar más detalladamente los resultados anteriores, en las gráficas 3 y 4 se

Tabla 8

Estadísticos AVR en el SEPP00

\begin{tabular}{|c|c|c|c|c|c|c|}
\hline \multicolumn{3}{|c|}{ Periodo } & $k$ & $\begin{array}{c}\text { Desviación } \\
\text { estándar }\end{array}$ & AVR & P-valor (\%) \\
\hline Completo & $03 / 01 / 1995$ & $31 / 12 / 2014$ & 3,1 & 0,4000 & $-1,3431^{* * *}$ & 0,4 \\
\hline 1 & $03 / 01 / 1995$ & $24 / 12 / 1996$ & 2,6 & 0,1855 & $0,6475^{* *}$ & 1,2 \\
\hline 2 & $26 / 12 / 1996$ & $21 / 12 / 1998$ & 1,5 & 0,3021 & 0,0636 & 56,2 \\
\hline 3 & $22 / 12 / 1998$ & $15 / 12 / 2000$ & 0,8 & 0,2060 & 0,0143 & 80,4 \\
\hline 4 & $18 / 12 / 2000$ & $19 / 12 / 2002$ & 0,5 & 0,2216 & $-0,0106$ & 86,0 \\
\hline 5 & $20 / 12 / 2002$ & $17 / 12 / 2004$ & 2,2 & 0,1846 & $-0,4324^{*}$ & 5,4 \\
\hline 6 & $20 / 12 / 2004$ & $14 / 12 / 2006$ & 1,8 & 0,1640 & 0,0943 & 33,0 \\
\hline 7 & $15 / 12 / 2006$ & $12 / 12 / 2008$ & 2,5 & 0,3733 & $-1,1036^{* * *}$ & 0,8 \\
\hline 8 & $15 / 12 / 2008$ & $10 / 12 / 2010$ & 2,1 & 0,2212 & $-0,3422$ & 13,2 \\
\hline 9 & $13 / 12 / 2010$ & $10 / 12 / 2012$ & 2,1 & 0,2974 & $-0,3442$ & 23,2 \\
\hline 10 & $11 / 12 / 2012$ & $31 / 12 / 2014$ & 1,6 & 0,2473 & 0,0223 & 78,8 \\
\hline
\end{tabular}

* Significativo al 10\%; ** significativo al 5\%; ** significativo al 1\%; k: periodo holding (ecuación [5]); P-valor: significancia del estadístico AVR.

Fuente: Elaboración de los autores a partir de MATLAB.

Tabla 9.

Estadísticos AVR en el DJIA

\begin{tabular}{|c|c|c|c|c|c|c|}
\hline \multicolumn{3}{|c|}{ Periodo } & $k$ & $\begin{array}{c}\text { Desviación } \\
\text { estándar }\end{array}$ & AVR & P-valor (\%) \\
\hline Completo & $\mathbf{0 2 / 0 1 / 1 9 9 5}$ & $\mathbf{3 0 / 1 2 / 2 0 1 4}$ & $\mathbf{3 , 0}$ & $\mathbf{0 , 3 6 1 3}$ & $-1,3320^{* * *}$ & $\mathbf{0 , 0}$ \\
\hline 1 & $03 / 01 / 1995$ & $02 / 01 / 1997$ & 2,4 & 0,1864 & $0,6221^{* *}$ & 1,8 \\
\hline 2 & $02 / 01 / 1997$ & $05 / 01 / 1999$ & 0,7 & 0,3114 & $-0,0129$ & 84,6 \\
\hline 3 & $05 / 01 / 1999$ & $05 / 01 / 2001$ & 0,9 & 0,2229 & 0,0556 & 52,2 \\
\hline 4 & $05 / 01 / 2001$ & $15 / 01 / 2003$ & 1,1 & 0,1889 & 0,0126 & 84,0 \\
\hline 5 & $15 / 01 / 2003$ & $19 / 01 / 2005$ & 2,0 & 0,2207 & $-0,2780$ & 17,0 \\
\hline 6 & $19 / 01 / 2005$ & $24 / 01 / 2007$ & 1,3 & 0,1882 & 0,0089 & 86,2 \\
\hline 7 & $24 / 01 / 2007$ & $27 / 01 / 2009$ & 2,6 & 0,3472 & $-1,1709 * * *$ & 0,4 \\
\hline 8 & $27 / 01 / 2009$ & $28 / 01 / 2011$ & 2,1 & 0,1883 & $-0,2523$ & 14,4 \\
\hline 9 & $28 / 01 / 2011$ & $04 / 02 / 2013$ & 2,0 & 0,3081 & $-0,2732$ & 27,8 \\
\hline 10 & $04 / 02 / 2013$ & $30 / 12 / 2014$ & 1,6 & 0,2219 & 0,0166 & 80,8 \\
\hline
\end{tabular}

* Significativo al 10\%; ${ }^{* *}$ significativo al 5\%; ${ }^{* *}$ significativo al 1\% k: periodo holding (ecuación [5]); P-valor: significancia del estadístico AVR.

Fuente: elaboración de los autores a partir de MATLAB. 
Estadístico AVR del indice SEP500 con intervalo de confianza al 95\%

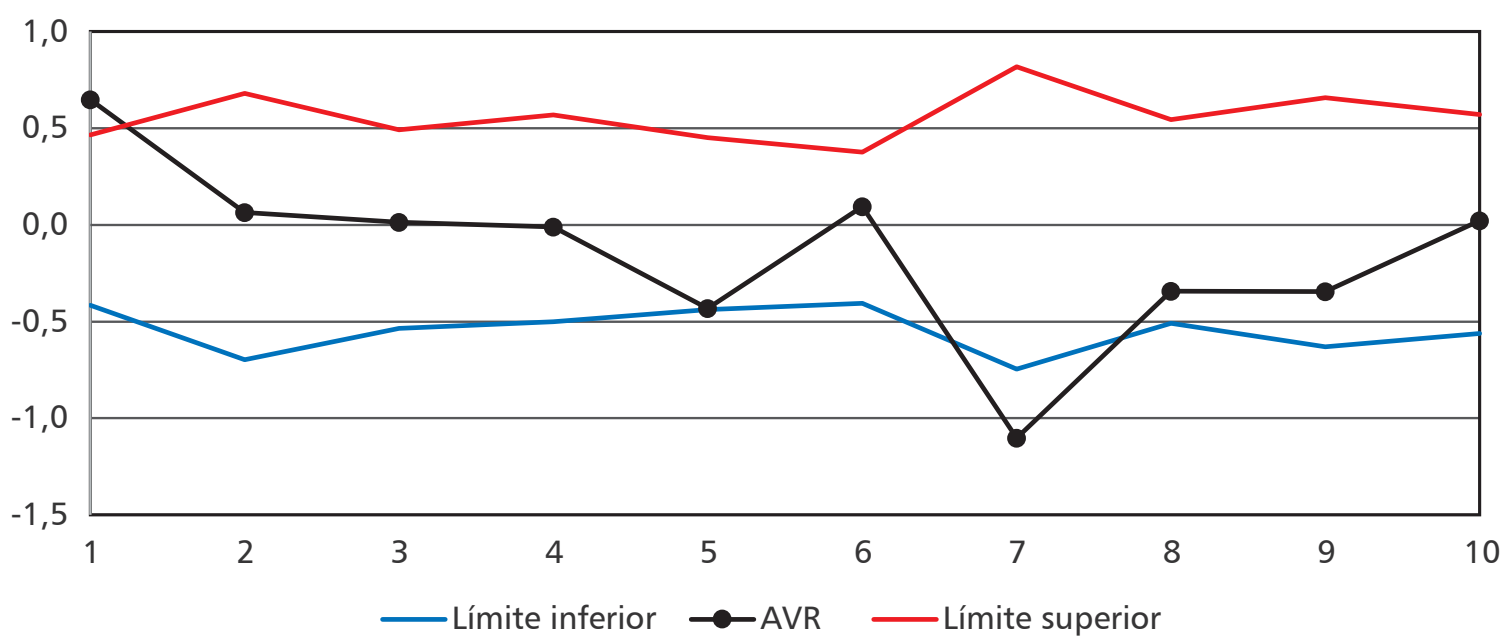

Nota: en la gráfica se muestran los valores del estadístico

AVR para diez subperiodos de tiempo (línea negra: subperiodos de la tabla 8) y los límites inferior y superior al $95 \%$ de confianza (líneas grises) para la serie del S\&P500.

Fuente: elaboración de los autores a partir de MATLAB.

Gráfica 4 .

Estadístico AVR del índice DJIA con intervalo de confianza al 95\%

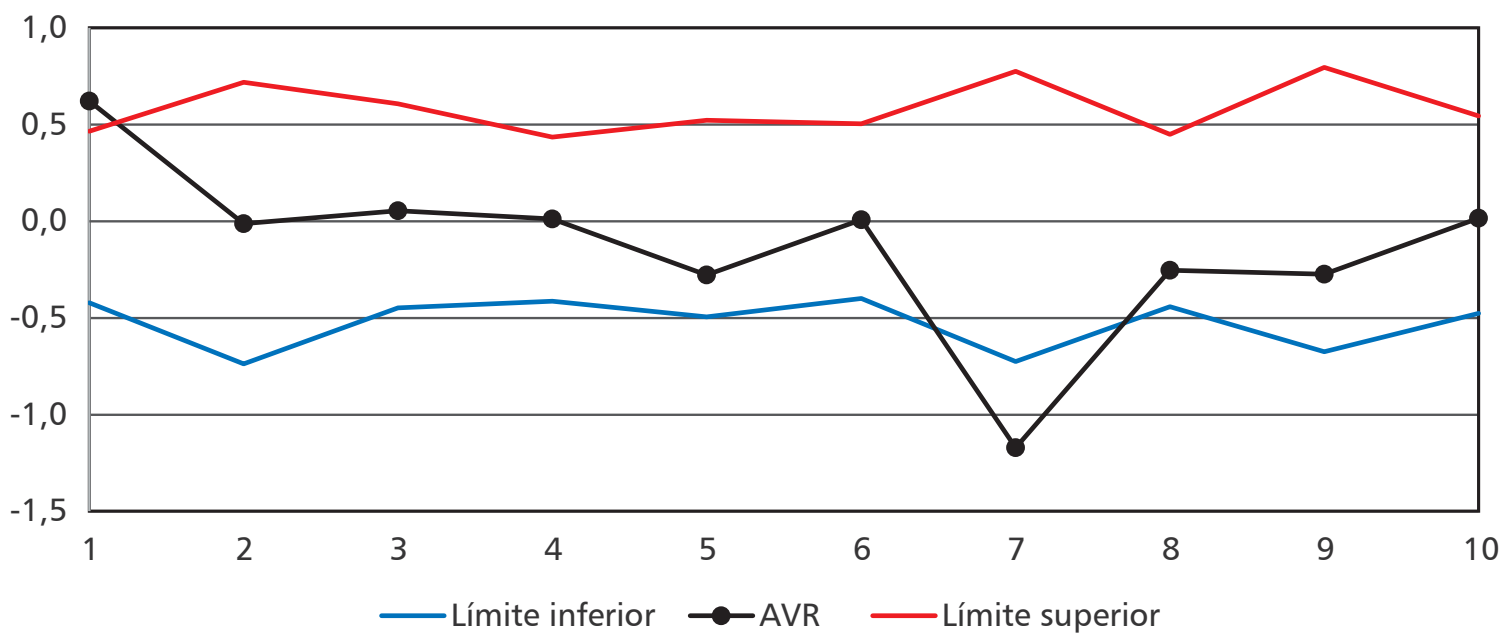

Nota: en la gráfica se muestran los valores del estadístico AVR para diez subperiodos de tiempo (línea negra: subperiodos de la tabla 9) y los límites inferior y superior al 95\% de confianza (líneas grises) para la serie del DJIA.

Fuente: elaboración de los autores a partir de MATLAB.

grafican los estadísticos AVR de los índices S\&P500 y DJIA, respectivamente.

Al observar las gráficas de los estadísticos AVR, puede evidenciarse que el comportamiento de ambos índices es similar. Por ello, se encuentra que en el mercado estadounidense, el nivel de correlación varía en el tiempo, de tal manera que hay cinco subperiodos con correlación serial positiva y cinco con correlación serial negativa en ambos índices; por lo tanto, podría afirmarse que la eficiencia del mercado estadounidense es dinámica, tal como lo postulan Kim, Shamsuddin 
y Lim (2011). Esto indicaría que a pesar de ser considerado un mercado eficiente, el nivel de eficiencia cambia en el tiempo, por cuanto tiene periodos aleatorios y periodos con patrones lineales que podrían permitir la predicción del mercado.

En cuanto a los intervalos de confianza, se observa que su amplitud no varía significativamente durante los diez subperiodos de estudio, lo que revelaría que el nivel de incertidumbre en el mercado se ha mantenido constante. Es importante destacar que en el séptimo subperiodo, que corresponde a los años de la crisis de la subprime (2006 a 2008), el estadístico AVR es negativo y estadísticamente significativo. En este sentido, Kim, Shamsuddin y Lim (2011) afirman que ello sería indicio de pánico y sobrerreacción por parte de los inversores en los mercados, comportamiento que es estudiado por la teoría de finanzas conductuales y no es contemplado dentro de los supuestos de eficiencia de los mercados.

Los anteriores resultados muestran la importancia de analizar la serie por subperiodos, ya que al evaluar la eficiencia en el periodo completo en los mercados objeto de estudio, se podría haber llegado a conclusiones erróneas.

\section{Análisis relativo entre mercados}

En la tabla 10 se presentan los resultados obtenidos al evaluar las series de los principales índices de Brasil, México y Estados Unidos, con el fin de realizar una comparación de eficiencia relativa.

Al comparar los resultados presentados en la tabla 10 , se destaca que el mercado con mayor nivel de aleatoriedad es Brasil, tanto en los subperiodos como en el periodo total. Además, el IBOVESPA es el único índice en el que no se rechaza la hipótesis de retornos independientes e idénticamente distribuidos durante el periodo completo mediante ambos test estadísticos. Es importante resaltar que contrario a la creencia general de que los mercados emergentes tienden a ser menos eficientes, los resultados de este trabajo muestran que en el mercado de Estados Unidos se rechaza con mayor frecuencia y significancia la hipótesis nula de aleatoriedad, en comparación con sus homólogos latinoamericanos.

Tabla 10.

Significancia de la aleatoriedad por periodos y subperiodos

\begin{tabular}{|c|c|c|c|c|c|c|c|c|c|}
\hline \multirow{2}{*}{\multicolumn{2}{|c|}{ Periodo (años) }} & \multicolumn{2}{|c|}{ IBOVESPA } & \multicolumn{2}{|c|}{ IPC } & \multicolumn{2}{|c|}{ S\&P500 } & \multicolumn{2}{|c|}{ DJIA } \\
\hline & & Rachas & AVR & Rachas & AVR & Rachas & AVR & Rachas & AVR \\
\hline & $\begin{array}{l}\text { ompleto } \\
995-2014)\end{array}$ & & & $* * *$ & & $* * *$ & $* * *$ & $* * *$ & $* * *$ \\
\hline 1 & (1995-1996) & & & $* * *$ & & & $* *$ & & ** \\
\hline 2 & (1997-1998) & $* * *$ & & $* * *$ & & & & & \\
\hline 3 & $(1999-2000)$ & & & $* * *$ & ** & & & & \\
\hline 4 & (2001-2002) & & & & & & & * & \\
\hline 5 & (2003-2004) & & & * & * & $* * *$ & * & $* * *$ & \\
\hline 6 & (2005-2006) & & * & & ** & ** & & & $* * *$ \\
\hline 7 & (2007-2008) & & & & & $* * *$ & $* * *$ & * & \\
\hline 8 & (2009-2010) & & & & * & * & & & \\
\hline 9 & $(2011-2012)$ & & & & & & & & \\
\hline 10 & $(2013-2014)$ & & & & * & ** & & ** & \\
\hline
\end{tabular}

* Rechazo de hipótesis nula de aleatoriedad significativo al 10\%; ** rechazo de hipótesis nula de aleatoriedad significativo al $5 \%$; ${ }^{* *}$ rechazo de hipótesis nula de aleatoriedad significativo al $1 \%$.

Fuente: elaboración de los autores. 
Adicionalmente, se encuentra que durante el periodo completo de estudio, todos los mercados presentan resultados que no son consistentes con el análisis por subperiodos. Por otra parte, se detecta que los patrones lineales aparecen en las series de Brasil y México antes del 2000, mientras que en Estados Unidos la mayoría se presenta entre 2003 y 2008.

\section{CONCLUSIONES}

Al evaluar los mercados de Brasil, México y Estados Unidos, mediante la prueba de rachas y el test ratio de varianza automático, en un periodo de veinte años y en subperiodos de dos años, se detectó que con un nivel de confianza del 95\%, podría concluirse que la eficiencia de los mercados latinoamericanos evaluados ha sido mayor que la del mercado estadounidense, representado por los índices S\&P500 y DJIA. Particularmente, se encuentra que la eficiencia de Estados Unidos habría disminuido desde el 2004, posiblemente a causa de la crisis de la subprime, mientras que la aleatoriedad de los índices bursátiles de Brasil y México aumentó desde el 2000, debido posiblemente a la mayor liquidez presentada en estos mercados, como consecuencia del aumento de la inversión extranjera en Latinoamérica, posibilitado en parte por el auge de la explotación de materias primas.

Por otra parte, partiendo de la significancia negativa del estadístico AVR, podría interpretarse que durante el periodo de crisis y poscrisis (2007 a 2010), en Estados Unidos habría existido pánico y sobrerreacción por parte de los inversores (Kim, Shamsuddin y Lim, 2011), los cuales son considerados sesgos de comportamiento desde la perspectiva de las finanzas conductuales. Se refuta de esta forma la hipótesis de mercado eficiente, dado que se niega la racionalidad de los inversores.

Es preciso señalar que en este trabajo, el mercado accionario de Estados Unidos es representado tanto por el índice S\&P500 como por el DJIA, y pese a sus diferentes composiciones, los resultados de las pruebas de eficiencia son similares para ambos índices. Ello muestra rechazo a la hipótesis de aleatoriedad en el periodo completo (1995 a 2014) y en los subperiodos de 2003 a 2005, de 2007 a 2008 (inicio de la crisis de la subprime) y de 2013 a 2014. Sin embargo, se resalta que la significancia de los estadísticos estimados para el DJIA es mayor que la del S\&P500, durante el periodo 2000-2012, es decir, para este periodo se podría inferir que el DJIA es más eficiente. Este hallazgo podría deberse a que el S\&P500 se compone de más sectores económicos y tamaños de empresa, que incluyen algunos activos no tan líquidos como los que componen el Dow Jones Industrial Average.

En cuanto a la revisión de la literatura se encuentran resultados mixtos en la aceptación o el rechazo de la hipótesis de eficiencia, lo cual podría ser explicado por el hecho de que los trabajos usan diferentes periodos de estudio. Al respecto, los resultados presentados en este trabajo revelan que en un mismo mercado se encuentra disparidad en cuanto a la evaluación de la eficiencia cuando se estudia el periodo completo de veinte años en comparación con los resultados obtenidos en el análisis por subperiodos. Se llega así a la conclusión de que es mejor analizar la serie por subperiodos durante un largo periodo de tiempo, puesto que permite evaluar la evolución de la eficiencia del mercado, tal como lo plantea Andrew Lo (2004) en su hipótesis de mercado adaptativo. En efecto, sería interesante investigar en futuros trabajos el impacto de la amplitud de los subperiodos en la evaluación de la eficiencia del mercado.

Finalmente, se aclara que los resultados de este estudio deben tomarse con precaución debido a que el test AVR evalúa la existencia de correlación serial en los índices, lo que podría interpretarse como un indicio de predictibilidad en la serie histórica; sin embargo, su uso sería muy limitado si se quisiera utilizar para predecir en tiempo real una posible crisis. De hecho, el propósito del test es detectar anormalidades en el mercado que podrían deberse pero no limitarse a crisis financieras. 


\section{REFERENCIAS}

1. Alexeev, V. y Tapon, F. (2011). Testing weak form efficiency on the Toronto Stock Exchange. Journal of Empirical Finance, 18(4), 661-691.

2. Brock, W., Lakonishok, J. y Lebaron, B. (1992). Simple technical trading rules and the stochastic properties of stock returns. The Journal of Finance, 47(5), 1752.

3. Butler, M. (2012). Computational intelligence for analysis concerning financial modelling and the adaptive market hypothesis (tesis doctoral). York: Universidad de York.

4. Choi, I. (1999). Testing the random walk hypothesis for real exchange rates. Journal of Applied Econometrics, 14(3), 293-308.

5. Comisión Económica para América Latina y el Caribe (Cepal) (2011). La inversión extranjera directa en América Latina y el Caribe 2010. Recuperado de http://www.cepal.org/publicaciones/xml/9/43289/2011322-LIE-2010-WEB_ULTIMO.pdf

6. Duarte, J. y Mascareñas, J. (2012). La eficiencia de los mercados de valores: una revisión. Análisis Financiero, 122, 21-35.

7. Duarte, J. y Mascareñas, J. (2014). Comprobación de la eficiencia débil en los principales mercados financieros latinoamericanos. Estudios Gerenciales, 122, 365-375.

8. Ely, R. A. (2011). Returns Predictability and Stock Market Efficiency in Brazil. Bras. Finanças, Rio de Janeiro, 9(4), 571-584.

9. Fama, E. (1965). The behavior of stock-market prices. The Journal of Business, 34-105.

10. Fernández, F. y González, C. (2000). Optimización de reglas técnicas en el IGBM usando algoritmos genéticos. Anales de Economía Aplicada. Oviedo.

11. Freitas, F. D., De Souza, A. F. y de Almeida, A. R. (2009). Prediction-based portfolio optimization model using neural networks. Neurocomputing, 72(10-12), 2155-2170.

12. Grieb, T. y Reyes, M. (1999). Random walk tests for Latin American equity indexes and individual firms. Journal of Financial Research, 22(4), 371-383.

13. Inghelbrecht, K., Heyman, D., Pauwels, S. y Marius, P. (2012). Technical trading rules in emerging stock markets. World Academy of Science, Engineering and technology, 59, 2241-2264.

14. Kim, J. (2009). Automatic variance ratio test under conditional heteroskedasticity. Finance Research Letters, 6(3), 179-185.

15. Kim, J., Shamsuddin, A. y Lim, K. (2011). Stock return predictability and the adaptive markets hypothesis: Evidence from century-long U.S. data. Journal of Empirical Finance, 18, 868-879.

16. Lo, A. (2004). The adaptive markets hypothesis: market eficiency from an evolutionary perspective. The Journal of Portfolio Management, 30(5), 15-29.

17. Lo, A. y MacKinlay, A. (1988). Stock market prices do not follow random walks: evidence from a simple specification test. Review of Financial Studies, 1(1), 41-66.

18. Lu, T., Shiu, Y. y Liu, T. (2012). Profitable candlestick trading strategies: The evidence from a new perspective. Review of Financial Economics, 21 (2), 63-68.

19. Ojah, K. y Karemera, D. (1999). Random walks and market efficiency test of Latin American emerging equity markets. The Financial Review, 34, 57-72. 
20. Shiller, R. (2003). From efficient markets theory to behavioral finance. Journal of Economic Perspectives, 17(1), 83-104.

21. Tabak, B. M. (2007). Testing for unit root bilinearity in the Brazilian stock market. Physica A: Statistical Mechanics and its Applications, 385(1), 261-269.

22. Urrutia, J. (1995). Tests of random walk and market efficiency for Latin American emerging equity markets. Journal of Financial Research, 299-309.

23. Valdivieso, R. (2004). Validación de la eficiencia y modelos de fijación de precios en el mercado mexicano de valores (tesis). México: Universidad Nacional Autonoma México. 
